\title{
Building Virtual Experiment Platform in the Curriculum of Network Operating System Using Virtual Machines
}

\author{
He Liu \\ School of Computer Science and Technology \\ HanKou University \\ Wuhan, China \\ e-mail:leuher@gmail.com
}

\author{
Runze Wan \\ College of Computer \\ Hubei University of Education \\ Wuhan, China \\ e-mail: name@xyz.com
}

\begin{abstract}
The curriculum of Network Operating System is one of the core courses in computer network specialty. According to the characteristics of the curriculum and cultivation target of the practical talents, this paper discusses how to use VMware in the teaching process to build the network teaching platform. Also, with the function of virtual machine in the snapshot, cloning, video recording and so on, it can ensure efficiency of the experimental teaching, and help students to realize the promotion from the verification of a single experiment to design their own complex network environment. Eventually, the Students can master server management and configuration, improve the ability of problem solving and analysis.
\end{abstract}

Keywords--virtual machine, VMware, experimental teaching, Windows network operating system

\section{INTRODUCTION}

"Windows network operating system" is one of major and professional courses in computer network technology specialty. The course includes tree main contents: Windows user management, Active Directory and Network Management. The course is an applied, practical, skilled, and highly integrated curriculum, focusing on the combination of theory and practice, requiring students to independently configure Windows systems and a variety of services. In the teaching process, the aims of this course include: the combination of theory and practice, self-learning ability, thinking ability and problem-solving skills. Students can engage in activities including construction, management and maintenance in computer networks, and the ability to practice basic skills, comprehensive technology applications and preliminary engineering design capability [1].

In the past few years, the authors used practice cases from enterprises to explain and demonstrate in teaching process. This method can make abstract and complex content becomes intuitive, and can help students to understand and master the specific configuration process. But some projects and experiments require student to build complex networks, therefore many problems exist: real host environment limits the number of people on the same machine, troubles on the physical connection. And, on more than one physical host and forth operation, experimental low efficiency; configuration process, if the student had a "traumatic" operation, it will need to spend a lot of time to return to normal state. Building a network environment using VMware virtual machines is a good solution to solve the above problems.

\section{WindOWS NETWORK OPERATING SYSTEM COURSES}

\section{A. Features of The Course}

The course emphasizes needs of the enterprises, centers on the professional competence and innovation. The course requires students to master the basic theories and configurations of Windows network operating system. It also requires students be proficient in various versions of Windows Server network operating system, can provide system management and services in a variety of common network servers, be able to understand and proficient in active Directory, have the abilities to analysis, design and implement the infrastructure of Windows server 2003/2008 platforms, be able to build an integrated network system. In general, the course has the following characteristics and requirements:

(1)The course requires solid theoretical foundations of network.

(2)The course requires students be proficient in the complex configuration of servers.

(3)There are lots of concepts, especially in active directory and user management.

(4)The course requires experience, error analysis and summarization in the configuration and application process.

\section{B. Main Contents of The Course}

The course of "Windows Network Operating System" includes three main contents, which are Windows user management, active directory, and network management [2].

The contents of Windows User Management include the profile and installation of Windows operating system, users management and groups management, NTFS file system, DFS distributed file system, registry, disk management, etc.

Microsoft Active Directory is a core component of Windows platforms, it is used to manage identification and relationship between various constituent elements in network environment and provide a powerful tool of configuration. The contents of Active Directory include the installation, active Directory domain, domain users, domain groups, and, etc. 
The contents Network management include the services like DHCP, DNS, WEB, FTP, PKI, IPSec, IP routing, NAT, VPN and, etc.

Moreover, the three main contents are highly contextual, such as configuration of DFS root in a distributed file system, can be divided into "domain root" and "independent root directory" types, it is a combination of user management with Active Directory content. Another example is the configuration of the terminal server, if it is in a domain environment, the permission setting of the domain users must consider the group permissions of the Remote Desktop Users, while considering the authority from remote server to the local server.

Therefore, this course requires students to have enough patience and time for repeated validations, debugging, and summarizations. If necessary, the course also need students to find solutions of problems through various channels.

\section{Difficulties and Emphases of The Course}

The course of "Windows network operating system" involves a very wide, heavy and difficult mainly in the following knowledge:

(1) The concepts of Active Directory, domains, domain trees, forests, WMI filters, organizational units, group policy, web sites, global catalog and, etc.

(2) Planning of the enterprise. Active Directory management. Domain Strategies includes: Migration, backup and recovery, monitor, and other integrated services. Backup and disaster recovery. Establishment of data backup / restore quality criteria, write a batch file backup and backup scripts, preparing a disaster recovery plan, analyze the reasons for the computer crashes and, etc.

(3) High-availability services. These jobs include: Managements of the server cluster, repairs of load balancing service in faulty networks, preparations of various hybrid cluster solutions and, etc.

(4) Network and communication services. For example, SNMP, VPN, routing and remote access, and, etc.

\section{The Shortcomings of Traditional Network Laboratory}

Traditional network laboratory in this course exhibited many shortcomings as follows:

(1) Difficulties in System Recovery. The processes of mastering the course's contents is gradual, students will inevitably make some mistakes at the beginning of the operations, and even the injury the machines. Typical operations of modifying and formatting the partitions on the line is inevitable when installing the systems. Some configuration errors basically can't be restored to its normal state, which affects the following students' operations.

(2) Recovery Card. Computers in lab rooms of universities are usually equipped with recovery card which can easily restore the system and configurations. But some kinds of recovery cards have limitations in Windows Server 2003, Vista, 2008 and other operating systems. In addition, the hard disk must be partitioned carefully when installing multi systems in the same disk. In this case, System Restore is very slow, the restore cards can't meet the needs of teaching.

(3) Verification experiments. Many experiments need lots of computers to be configured in the same time while students just need to verify the results. It is difficult for students to have independent thinking of designing their own experiments.

(4) Limited experiment conditions. The traditional experimental environment due to hardware limitations, students can only be in the room with the completion of the experimental design, it is difficult for students to do extracurricular experiments by self. Limitations of hardware conditions can frustrate the students, and keep them away from the labs. Some complex experiments, such as routing and remote access, DHCP proxy, WINS Proxy, etc. need multiple network segments, must be changed in a professional lab.

\section{EXPERIMENT PLATFORM USING VMWARE}

Building the network environment is the foundation job of the experiment in the course, it is very complicated and will consume a lot of time. VMware virtualization will be able to achieve network experiments on a single computer, help building the network quickly, help recovering from system failures. Students can devote more to important experimental session [3, 4].

\section{A. Network Environment of Multiple Segments}

Many experiments in the course need to set up at least two segments of the network structure, if the experiments are carried out using real computers and network devices, schools need to purchase lots of computers, switches and routers. VMware virtualization platform can be easily implemented across experiment the network.

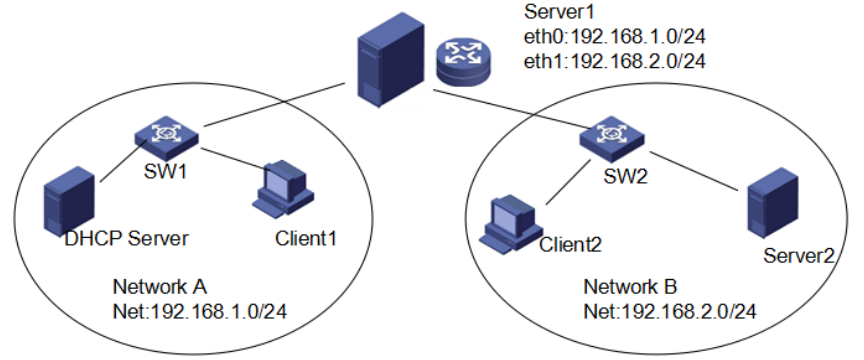

Fig. 1 a network structure of multi-segments

In the network environment shown in Figure 1, Server1 and Client1 is connected to the switch SW1, which is located in a network segment; while Client2 and Server2 is connected to the switch SW2, located on another network segment. The router Router1 has two network interfaces, eth0 is connected to the switch SW1, eth1 is connected to the switch SW2. VMware provides up to 10 virtual switches, in addition to VMnet0 and VMnet8 to bridge mode and NAT mode, the other virtual switch works in Host-only mode. Students can use these virtual switches to create soft routers and to achieve multi-segment in the experiments. 
There are two steps to configure a connected network using VMware. First configuring the virtual interfaces as switches shown in Figure 2 correctly according to the topology.

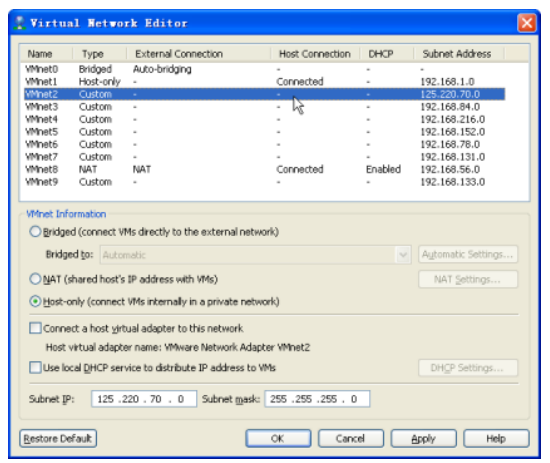

Fig. 2 Virtual Network Editor

Secondly configuring the virtual machines connecting to virtual switches like the Figure 3 . It is easy to configure a windows server 2008 as a soft router to connect different segment of networks.

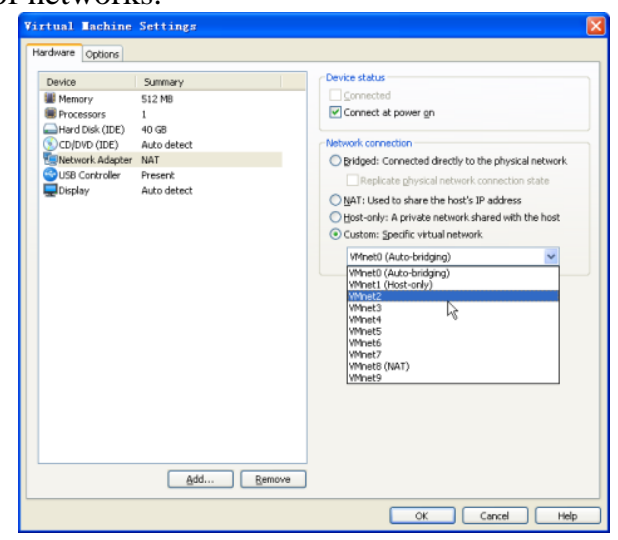

Fig. 3 connecting a virtual machine to the virtual switch

Multi-segment network environment is needed in some complex experiments, such as the DHCP relay agent, WINS Proxy, Routing and Remote Access. Some experiments, such as RIP, NAT, VPN, need three or even more network segments. In these situations, this approach still works by increasing the numbers of routers, computers and virtual switches.

\section{B. Advantages of VMware in Experiments}

(1) The installation of the operating system is the basic content in the course, students should be familiar with different types of installation and basic configuration of different versions of operating system. Errors may occur when students install systems on physical computers directly, and it will lead to data loss, or even system can't load and boot properly. Students can freely to partition, format and execute other operations, and can easily add more virtual hard disks and virtual devices without worrying about the failures and damages of the devices.

(2) It is easy to build a complex network environment by VMware virtualization. It can meet the requirement of achieving a multiple network architecture, meet the needs of DHCP relay agent, VPN, routing and remote access and so on.

(3) Snapshot is powerful function in the lab to configure a variety of network environments. Snapshots can store different states of the computer at any time, if problems and errors occur during the experiment. System can easily be restored to the state before the wrong operations.

(4) The team function of VMware can divide virtual machines into groups for centralized management. It can simultaneously open and close the virtual machines in the same group, or set up a group of virtual machines boot delay to avoid overloading the host CPU. Moreover, it is easy to simulate bandwidth and packet loss in the experiments by using virtual switch Team. For example, it can limit the bandwidth between $1 \mathrm{~K} \sim 1 \mathrm{G}$, and simulate the packet loss from $0 \%$ to $100 \%$. Thumbnail images from the Team display the states of all virtual machines, and can make a global view of the experiment.

\section{Preparation of The Network Environment}

Each student usually needs an experiment environment with at least five computers of different operation systems to achieve a complex experiment. Teacher can configure different virtual computers, define the roles of computers in the experiment, and then make snapshots in advance. For example, teacher can deploy five virtual machines named Server1, Server2, Router, Client1 and Client2, the servers and router can be installed windows server 2008, and the clients may be installed with windows 7 . All of the virtual machines have just a snapshot without IP address, workgroup and domain information. The initialization of the experiment is already done, and the students just need to the experiment according to the handbook.

In addition, a handbook of experiment has the full information of the virtual experiment lab including a detailed network topology, the use of the virtual machine, the instruction of snapshots, roles of virtual machines, documents of configurations. All these make easy for students to improve the efficiency of the unit hours in the lab.

Restore cards are managed in manual restore mode, as far as possible to ensure the continuity of the experiment, while reducing the impact on the students in the same laboratory.

\section{CONCLUSIONS}

As a very practical course, the Network Operating System requires students to do lots of experiments, no just relying on teachers' teaching and practical demonstration of the theory, especially in the part of the Active Directory. It is difficult to truly understand the essence of the Windows platform for network management without lots of practice. The network environment to build and customize the network configuration of each computer role, need students to have administrator thinking and engineering technical ability. Building a network platform using VMware allows students to quickly build their own desired network 
environment without worrying about errors caused by the wrong operations in the real environment, and the students have no much psychological burden. All these will help students to play the full role of motivation, and help to improve the efficiency of the experiments.

\section{ACKNOWLEDGMENT}

This paper is supported by Foundation of Hubei (No. B20128012).

\section{REFERENCES}

[1] RuiJin Wang,etc. a brief discussion about Development and construction On the "Network Operating System" course [J], Xinjiang Vocational University, October 2008, Vol 16, No. 5, 43-45.

[2] Lan $\mathrm{Hu}$, New features of Windows Server 2008 [J], journal of Software Guide, May 2009, 47 - 50.

[3] Xiangying Zhou, Visualization experiment platform of computer networks based on VMware virtual technology [J]. Laboratory Research and Exploration, 2006 (7).

[4] HaiJing Bao, etc., Researches on virtual experiment platform based on VMWare Virtual Network Laboratory [J], Computer Technology and Development, June 2010, 242-245. 